PROCEEDINGS OF THE

AMERICAN MATHEMATICAL SOCIETY

Volume 28, No. 1, April 1971

\title{
A RIEMANN MAPPING THEOREM FOR $C(X)$
}

\author{
HUGH E. WARREN
}

Abstract. Given a strong definition of "conformal map," there is a class of domains in $C(X)$ conformally equivalent to the open unit ball which are in a specific way "interiors" of curves.

1. Introduction. In 1943 E. R. Lorch [5] extended the idea of "holomorphic function" to commutative Banach algebras with identity. Briefly, if $A$ denotes the algebra and $f$ is a mapping from $A$ to $A$, then $f$ is called holomorphic at a point $a \in A$ if there is an algebra element $f^{\prime}(a)$ such that for $h \in A$ we have

$$
\left\|f(a+h)-f(a)-h f^{\prime}(a)\right\|=o(\|h\|)
$$

as $\|h\|$ tends to zero. A mapping is holomorphic on an open set if it is holomorphic at each point of the set. Lorch and others have shown that many classical theorems of complex function theory carry over to this more general setting. The Riemann mapping theorem cannot be extended without some qualification.

Two open sets in a commutative Banach algebra will be called "conformally equivalent" if there is a one to one mapping between them which is holomorphic in both directions. A first try at a Riemann mapping theorem might be that the open unit ball of the algebra is conformally equivalent to every bounded homeomorph of itself. To this B. W. Glickfeld gives the following counterexample $[2, \mathrm{p} .45]$. In the algebra $C([0,1])$ the set of functions $f$ such that $|f(x)|<1$ for $0 \leqq x \leqq 1 / 2$ and $|f(x)|<2$ for $1 / 2<x \leqq 1$ is not conformally equivalent to the open unit ball. This set is a homeomorph of the open unit ball under the mapping $f \rightarrow g$ defined by

$$
\begin{aligned}
& g(x)=f(x), \quad|f(x)| \leqq m, \\
& =e^{i \theta}[(1-m) r+m] /(2-m), \quad \text { otherwise, }
\end{aligned}
$$

where $m=\max \{|f(x)|: 0 \leqq x \leqq 1 / 2\}$ and $f(x)=r e^{i \theta}$.

A class of domains conformally equivalent to the open unit ball of the algebra will be described below. This class develops from Lorch's extended notion of the interior of a curve. First, a "simple closed curve" in an algebra $A$ will be a continuous map $\Gamma$ of the unit circle

Received by the editors March 20, 1969.

AMS 1969 subject classifications. Primary 4655, 3040; Secondary 4625.

Key words and phrases. Riemann mapping, conformal mapping, interior of a curve, function algebra.

Copyright () 1971, American Mathematical Society 
into $A$ with the strong property: if $M$ is any nonzero algebra homomorphism of $A$ into the complex numbers, then $M \circ \Gamma$ is a homeomorphism. In this definition the curve is the mapping rather than the image. The image of each $M \circ \Gamma$ is a Jordan curve in the plane. Second, the "interior of $\Gamma$ " is defined to be all elements $a \in A$ such that $M(a)$ is interior to the image of $M \circ \Gamma$ in the usual sense for every $M$ as above. It is of course not evident that interior elements exist. Later on $I$ will use the fact that the interior of $\Gamma$ is an open set. This fact follows from the observation that if $a$ is in the interior of $\Gamma$ then the distance from $M(a)$ to the image of $M \circ \Gamma$ is a continuous positive function of $M$, that is, continuous on the maximal ideal space. The maximal ideal space is compact, so there is a minimal distance $d>0$. If $\|b-a\|<d$, then $b$ also is in the interior of $\Gamma$.

The purpose of this paper is to prove

TheOREM A. If $X$ is a compact Hausdorff space and $\Gamma$ is a simple closed curve in $C(X)$, then the interior of $\Gamma$ is conformally equivalent to the open unit ball of $C(X)$.

In $\$ 5$ it is shown that the classical theorem on extension of the conformal map to the boundary also generalizes.

I list here some notational conventions. The letter $A$ and the word "algebra" will be used in place of "commutative complex Banach algebra with identity." The complex plane $C$ will be identified with the complex multiples of the identity. The letter $K$ will stand for the open unit disc in $C$. The unit circle, which is the boundary of $K$, will be written $T$ and points on $T$ will be written $e^{i \theta}$. The letter $M$ will always stand for a nonzero homomorphism of $A$ into $C$. A curve or path will always be identified with a parametrization of the curve or path.

2. The mapping function. Integrals along rectifiable paths in an algebra $A$ are defined in a manner strictly analogous to the definition of complex integrals as limits of Riemann sums. One proves just as in the classical case

Lemma 1. Let $D$ be an open set in $A$ and $\Gamma$ a rectifiable path in $A$ such that when $a \in \Gamma$ and $\alpha \in D$, then $a-\alpha$ is invertible. Let $g$ be an $A$-valued function defined and continuous on $\Gamma$. Then the function $f: D \rightarrow A$ defined by $f(\alpha)=(2 \pi i)^{-1} \int_{\mathrm{r}} g(a)(a-\alpha)^{-1} d a$ is holomorphic.

As a special case, let $I$ be the identity parametrization of the unit circle in $A$. This parametrization defines a rectifiable simple closed curve. The image of $M \circ I$ is always $T$, so that the interior of $I$ is the spectral open unit ball of $A$, which we shall call $S$. For $e^{i \theta} \in T$ and 
$\alpha \in S$ we know that $e^{i \theta}-\alpha$ is invertible. Thus for any continuous map $g: T \rightarrow A$ we define a holomorphic function $\Phi_{v}: S \rightarrow A$ by

$$
\Phi_{g}(\alpha)=(2 \pi i)^{-1} \int_{I} g\left(e^{i \theta}\right)\left(e^{i \theta}-\alpha\right)^{-1} d e^{i \theta} .
$$

Consider a simple closed curve $\Gamma: T \rightarrow A$. Since $\Gamma$ is continuous, we can form $\Phi_{\Gamma}$. Under special conditions $\Phi_{\Gamma}$ is a conformal equivalence between $S$ and the interior of $\Gamma$.

Look first at the case $A=C$. Here $\Gamma$ is a continuous complex valued function on $T$, and $\Phi_{\Gamma}$ is an ordinary holomorphic function on $K$. Write $\Gamma \in H$ if $\Gamma$ has a continuous extension to the closed unit disc which is holomorphic on $K$. If $\Gamma \in H$, then $\Phi_{\Gamma}$ is that extension to $K$ and the function values $\Phi_{\Gamma}\left(r e^{i \theta}\right)$ converge uniformly in $\theta$ to $\Gamma\left(e^{i \theta}\right)$ as $r$ tends to one. This convergence property and the interior mapping theorem show that $\Phi_{\Gamma}$ maps $K$ onto the interior of $\Gamma$. Since $\Gamma\left(e^{i \theta}\right)$ goes around the interior of $\Gamma$ just once as $\theta$ goes from zero to $2 \pi$, the principle of the argument shows that $\Phi_{\Gamma}$ is one to one. Thus $\Phi_{\Gamma}$ is a conformal mapping of $K$ onto the interior of $\Gamma$. Even if $\Gamma$ is not in $H$, the ordinary Riemann mapping theorem says there is some conformal map $f(z)$ of $K$ onto the interior of $\Gamma$. Whenever a simply connected domain in $C$ is bounded by a Jordan curve any corresponding Riemann mapping function extends to a homeomorphism of the boundaries $\left[4\right.$, p. 367]; therefore $f\left(r e^{i \theta}\right)$ converges uniformly in $\theta$ to a homeomorphism $f\left(e^{i \theta}\right)$ of $T$ onto the image of $\Gamma$. We see that any $\Gamma$ has at least a reparametrization $f \in H$ such that $\Phi_{f}(z)=f(z)$ is a conformal mapping of $K$ onto the interior of $\Gamma$.

Return to a general algebra. Say that a simple closed curve is $H$ if its composition with any functional $M$ is in $H$. Look at $M \circ \Phi_{\mathrm{r}}(S)$ when $\Gamma$ is an $H$ simple closed curve. We recall that the in tegral which defines $\Phi_{\Gamma}(\alpha)$ is a limit in the algebra norm of Riemann sums. Applying one of the algebra homomorphisms $M$ to these sums and passing to the limit, we get $M \circ \Phi_{\Gamma}(\alpha)=\Phi_{M \circ \Gamma}(M(\alpha))$. Now $\Phi_{M \circ \Gamma}$ is a conformal mapping of $K=M(S)$ on to the interior of $M \circ \Gamma$, because $M \circ \Gamma \in H$. It follows that $\alpha \in S$ implies that $M \circ \Phi_{\Gamma}(\alpha)$ is in the interior of $M \circ \Gamma$ for every $M$. That is to say, $\Phi_{\Gamma}(S)$ lies in the interior of $\Gamma$.

Supposing still that $\Gamma$ is an $H$ simple closed curve, we look for an inverse to $\Phi_{\Gamma}$. The function $\Gamma^{-1}: \Gamma(T) \rightarrow T$ is continuous, for $\Gamma$ is continuous and one to one on the compact set $T$. Also if $a \in \Gamma$ and $\beta$ is in the interior of $\Gamma$, which is open, then $a-\beta$ is invertible. Assuming that $\Gamma$ is rectifiable, we can apply Lemma 1 and produce a holomorphic function 


$$
\psi_{\Gamma}(\beta)=(2 \pi i)^{-1} \int_{\Gamma} \Gamma^{-1}(a)(a-\beta)^{-1} d a,
$$

which maps the interior of $\Gamma$ into $S$. For all $M, M \circ \Phi_{\Gamma} \circ \psi_{\Gamma}(\beta)=M(\beta)$, so if $A$ is a semisimple algebra we can assert that $\Phi_{\Gamma}$ and $\psi_{\Gamma}$ are inverse to one another. In this case $\Phi_{\Gamma}$ is a conformal equivalence between $S$ and the interior of $\Gamma$. In other words, in a semisimple algebra the interior of a rectifiable $H$ simple closed curve is conformally equivalent to the open unit ball.

3. Curves in $C(X)$. If a simple closed curve $\Gamma$ is not $H$, then the mapping $\Phi_{\Gamma}$ appears to be useless. It is heartening, however, that two distinct curves in an algebra can have the same interior. To prove Theorem A we shall prove

ThEOREM B. If $X$ is a compact Hausdorff space, then every simple closed curve in $C(X)$ has the same interior as some $H$ simple closed curve.

In the proof below let $\Gamma$ be the simple closed curve. For each $\theta$, $\Gamma\left(e^{i \theta}\right)$ is a function, whose value at a point $x \in X$ will be denoted $\Gamma\left(e^{i \theta}, x\right)$. The nonzero complex homomorphisms of $C(X)$ amount to point evaluation. Consequently, for each fixed $x, \Gamma\left(e^{i \theta}, x\right)$ is a homeomorphism in $e^{i \theta}$ of the unit circle into the complex plane. The image curve has an $H$ parametrization $F\left(e^{i \theta}, x\right)$ which is uniquely determined by the condition $F\left(e^{i \theta}, x\right)=\Gamma\left(e^{i \theta}, x\right), \theta=0, \pi / 2, \pi$. To show this uniqueness, observe that a conformal mapping of the complex unit disc onto itself is determined by the images of any three boundary points. For a fixed $\theta, F\left(e^{i \theta}, x\right)$ gives a function $F\left(e^{i \theta}\right)$ on $X$. The proof will show that $F\left(e^{i \theta}\right) \in C(X)$, that as a mapping of $T$ into $C(X), F$ is continuous, and that in fact $F$ is an $H$ simple closed curve whose interior coincides with that of $\Gamma$.

Fix a point $x_{0} \in X$. Since the unit circle is compact and since $\Gamma: T \rightarrow C(X)$ is continuous, the family of functions $\left\{\Gamma\left(e^{i \theta}\right): 0 \leqq \theta<2 \pi\right\}$ is equicontinuous at $x_{0}$. That is, given a $\delta>0$, there is a neighborhood $U$ of $x_{0}$ such that if $x \in U$ then for all $\theta$

$$
\left|\Gamma\left(e^{i \theta}, x_{0}\right)-\Gamma\left(e^{i \theta}, x\right)\right|<\delta .
$$

Let $\epsilon>0$ be given. It is possible to choose the $\delta$ above so that for all $x \in U$ and all $\theta$

$$
\left|F\left(e^{i \theta}, x_{0}\right)-F\left(e^{i \theta}, x\right)\right|<\epsilon .
$$

For suppose not. There are in that case positive numbers $\delta_{n}$ tending to zero and a sequence of points $x_{n}$ in $X\left(x_{n}\right.$ in the $U$ found above for 
$\left.\delta=\delta_{n}\right)$ such that if we put $g_{n}\left(e^{i \theta}\right)=\Gamma\left(e^{i \theta}, x_{n}\right)$ and $f_{n}\left(e^{i \theta}\right)=F\left(e^{i \theta}, x_{n}\right)$, $n=0,1, \cdots$, then $\left\|g_{0}-g_{n}\right\|_{u}<\delta_{n}$ and $\left\|f_{0}-f_{n}\right\|_{u} \geqq \epsilon$. Here $\|h\|_{u}$ $=\sup _{\theta}\left|h\left(e^{i \theta}\right)\right|$. For $n \geqq 0, g_{n}(T)$ is a Jordan curve in the plane which bounds a simply connected domain $D_{n}$. Because $\left\|g_{0}-g_{n}\right\|_{u} \rightarrow 0, D_{0}$ and all but finitely many $D_{n}$ contain a common point, which without loss of generality we take to be zero. For $n=0,1, \cdots$, let $\varphi_{n}(z)$ be the conformal mapping of $K$ onto $D_{n}$ such that $\varphi_{n}(0)=0$ and $\varphi_{n}^{\prime}(0)$ $>0$. Since $D_{n}$ is the interior of a Jordan curve, $\varphi_{n}$ extends to a homeomorphism of $K^{-}$onto $D_{n}^{-}$. It is a result of $\mathrm{R}$. Courant that the mappings $\varphi_{n}$ converge uniformly on the closed unit disc to $\varphi_{0}[1$, Theorem IIIa]. Courant's hypothesis is that the boundary curves given by the $g_{n}$ converge uniformly to the curve given by $g_{0}$ in the following sense $[1$, p. 106]:

Definition. Es seien $\Gamma_{1}, \Gamma_{2}, \Gamma_{3}, \ldots$ unendlich viele Jordansche Kurven und es existiere eine Jordansche Kurve $\Gamma$, die identisch ist mit der Menge aller Punkte, welche einen mit wachsendem $n$ gegen Null konvergierenden Abstand von $\Gamma_{n}$ besitzen. Dann sagen wir, daß die Kurven $\Gamma_{n}$ gleichmäßig gegen $\Gamma$ konvergieren wenn folgende Bedingung erfüllt ist: Ist $P$ ein Punkt auf $\Gamma$ und sind $P_{1}, P_{2}$ zwei Punkte innerhalb $\Gamma$, deren Abstand von $P$ kleiner als $\epsilon$ ist, so lassen sich für hinreichend großes $n$ die Punkte $P_{1}$ und $P_{2}$ durch einen ganz innerhalb $\Gamma_{n}$ liegenden Streckenzug verbinden, dessen Länge unterhalb einer von $n, P_{1}$ und $P_{2}$ unabhängigen, mit $\epsilon$ gegen Null konvergierenden Schranke bleibt. ${ }^{1}$

Let $\Gamma_{n}$ be the curve parametrized by $g_{n}$ and $\Gamma$ by $g_{0}$. The condition $\left\|g_{0}-g_{n}\right\|_{u} \rightarrow 0$ implies that $\Gamma_{n}$ converges uniformly to $\Gamma$ in the sense above. This is perhaps what the footnote alludes to.

Recall that, for each $n, f_{n}\left(e^{i \theta}\right)$ gives the boundary values of the conformal mapping $f_{n}(z)$ of $K$ onto $D_{n}$ which is determined by $f_{n}\left(e^{i \theta}\right)$ $=g_{n}\left(e^{i \theta}\right), \theta=0, \pi / 2, \pi$. It follows that the functions $h_{n}=\varphi_{n}^{-1} \circ f_{n}$ map $K$ conformally onto itself and satisfy $h_{n}\left(e^{i \theta}\right) \rightarrow h_{0}\left(e^{i \theta}\right)$ for $\theta=0, \pi / 2, \pi$.

LEMMA 2. If functions $h_{n}$ map the unit disc conformally onto itself and converge at three points on the unit circle, then they converge uniformly on the closed unit disc.

Proof. Assume without loss of generality that the $h_{n}$ converge at $1, i$ and -1 to the identity map. For otherwise choose fractional linear transformations $u$ and $v$ such that the sequence $u \circ h_{n} \circ v$ meets the assumption and use the uniform continuity of $u$ and $v$

${ }^{1}$ Es ist leicht, diese Definition ihrer Beziehung auf das Innere der Kurven zu entkleiden. 
on the closed disc. By the Schwarz Lemma $\left[4\right.$, p. 236] $h_{n}(z)$

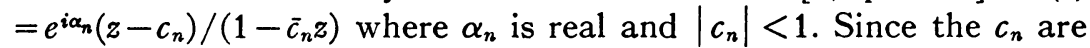
bounded, we have

$2\left(e^{i \alpha_{n}}-1\right)$

$$
\begin{aligned}
= & \left(h_{n}(1)-1\right)\left(1-\bar{c}_{n}\right)-\left(h_{n}(-1)+1\right)\left(1+\bar{c}_{n}\right) \rightarrow 0, \\
e^{i \alpha_{n}\left(1+i-2 c_{n}\right)-} & (1+i) \\
& =\left(h_{n}(1)-1\right)\left(1-\bar{c}_{n}\right)+\left(h_{n}(i)-i\right)\left(1-i \bar{c}_{n}\right) \rightarrow 0 .
\end{aligned}
$$

We read off successively that $e^{i \alpha_{n}}$ tends to 1 and that $c_{n}$ tends to zero. Since $|z| \leqq 1$ implies $\left|z-h_{n}(z)\right| \leqq\left|1-e^{i \alpha_{n}}\right|+2\left|c_{n}\right| /\left(1-\left|c_{n}\right|\right)$, we are done.

It now follows that the functions $f_{n}=\varphi_{n} \circ h_{n}$ converge uniformly to $f_{0}=\varphi_{0} \circ h_{0}$; thus we cannot have $\left\|f_{0}-f_{n}\right\|_{u} \geqq \epsilon$ for all large $n$. Now fix $\theta$ and let $x_{0}$ vary over $X$. One reads off from $\left({ }^{*}\right)$ that $F\left(e^{i \theta}, x\right)$ is a continuous function of $x$.

Again let $\epsilon>0$ be given. For each $x_{0} \in X$ let $U_{x_{0}}$ be a neighborhood of $x_{0}$ such for $x \in U_{x_{0}}$ the inequality $\left(^{*}\right)$ holds. The space $X$ is compact, so choose points $x_{1}, \cdots, x_{n}$ such that $X=U_{x_{1}} \cup \cdots \cup U_{x_{n}}$. Recall that, for each $k, F\left(e^{i \theta}, x_{k}\right)$ is continuous in $\theta$. Fix a real number $\varphi$ and choose neighborhoods $V_{1}, \cdots, V_{n}$ of $\varphi$ so that $\theta \in V_{k}$ implies

$$
\left|F\left(e^{i \theta}, x_{k}\right)-F\left(e^{i \varphi}, x_{k}\right)\right|<\epsilon .
$$

Put $V=V_{1} \cap \cdots \cap V_{n}$. Choose any $\theta \in V$ and let $x$ be an arbitrary point of $X$. The point $x$ lies in some neighborhood $U_{x_{k}}, 1 \leqq k \leqq n$. Adding and subtracting $F\left(e^{i \varphi}, x_{k}\right)$ and $F\left(e^{i \theta}, x_{k}\right)$ and expanding via the triangle inequality we see that

$$
\left|F\left(e^{i \varphi}, x\right)-F\left(e^{i \phi}, x\right)\right|<3 \epsilon \text {. }
$$

That is, as functions of $x, F\left(e^{i \varphi}, x\right)$ and $F\left(e^{i \theta}, x\right)$ are uniformly closed. The positive number $\epsilon$ is arbitrary; therefore $F$ maps $T$ continuously into $C(X)$.

To finish the proof of Theorem B we need only refer to the definitions of " $H$ curve" and "interior," keeping in mind that the functionals $M$ are evaluation at points of $X$.

4. Nonrectifiable curves. If the curve $F$ constructed above is rectifiable we can construct the inverse mapping $\psi_{F}$ as in $\$ 2$ and conclude immediately that $\Phi_{F}$ is a conformal equivalence between the interior of $F$, which is the interior of $\Gamma$, and the open unit ball of $C(X)$.

If $F$ is not rectifiable define $\psi_{F}$ as follows. Let $g$ be a function in the 
interior of $F$. Define the function $h=\psi_{F}(g)$ on $X$ by letting $h(x)$ be the inverse image of $g(x)$ under the conformal mapping of $K$ which converges to the boundary mapping $F\left(e^{i \theta}, x\right)$. We have $h \in C(X)$. For suppose to the contrary that $h$ is not continuous at $x_{0}$. For $n=1$, $2, \cdots$ choose a neighborhood $U_{n}$ of $x_{0}$ such that $\left(^{*}\right)$ holds with $\epsilon=1 / n$. Since $h_{n}$ is not continuous at $x_{0}$ we can find an $\eta>0$ and for each $n$ a point $x_{n} \in U_{n}$ such that $\left|h\left(x_{0}\right)-h\left(x_{n}\right)\right| \geqq \eta$. Let $f_{n}$ be the conformal mapping of $K$ determined by $f_{n}\left(e^{i \theta}\right)=F\left(e^{i \theta}, x_{n}\right), n \geqq 0$. By the maximum principle $\left|f_{0}(z)-f_{n}(z)\right|<1 / n$ for $|z| \leqq 1$. The numbers $h\left(x_{n}\right)$ lie in $K$ and have a limit point $y \neq h\left(x_{0}\right)$ in $K^{-}$. Pass to a subsequence so that $h\left(x_{n}\right) \rightarrow y$. Remembering that $f_{n}\left(h\left(x_{n}\right)\right)=g\left(x_{n}\right)$, we see that, for each $n$,

$$
\begin{aligned}
\left|f_{0}(y)-f_{0}\left(h\left(x_{0}\right)\right)\right| \leqq & \left|f_{0}(y)-f_{0}\left(h\left(x_{n}\right)\right)\right| \\
& +\left|f_{0}\left(h\left(x_{n}\right)\right)-f_{n}\left(h\left(x_{n}\right)\right)\right|+\left|g\left(x_{n}\right)-g\left(x_{0}\right)\right| .
\end{aligned}
$$

Since $f_{0}$ is continuous at $y$ and $g$ is continuous at $x_{0}$, the right-hand side of the inequality goes to zero as $n$ increases. Hence $f_{0}(y)=f_{0}\left(h\left(x_{0}\right)\right)$. But that is impossible because $f_{0}$ is one to one on $K^{-}$.

At this point we have a mapping $\psi_{F}$ from the interior of $F$ into $S$, the open unit ball of $C(X)$. Clearly $\Phi_{F}$ and $\psi_{F}$ are inverse to each other; therefore $\Phi_{F}$ maps $S$ onto the interior of $F$. It is shown by Glickfeld that if a one to one holomorphic function on a domain in $C(X)$ has open range then the inverse mapping is holomorphic. Apply this result to $\Phi_{F}$. We see that $\Phi_{F}$ is a conformal equivalence between $S$ and the interior of $F$. In view of the construction of $F$ from $\Gamma$, Theorem $\mathrm{A}$ is proved.

5. Correspondence of the boundaries. Let $F$ be an $H$ simple closed curve in $C(X)$. For each $x \in X$ let $F(z, x)$ be the function continuous on $K^{-}$and conformal on $K$ which agrees with $F\left(e^{i \theta}, x\right)$ on $T$. If $\Phi_{F}$ has a continuous extension to the closure $S^{-}$of the unit ball, then the continuity of the point evaluation functionals requires that $g=\Phi_{F}(h)$ be given by $g(x)=F(h(x), x)$ for all $h \in S^{-}$. We show now that $g \in C(X)$ and that the extended mapping $\Phi_{F}$ is continuous on $S^{-}$.

For a fixed $x, F(z, x)$ is a uniformly continuous function on the compact set $K^{-}$. Using relation $\left({ }^{*}\right)$, the maximum principle for analytic functions and the compactness of $X$, one proves by standard methods that there is a single $\delta=\delta(\epsilon)$ such that $z, w \in K^{-}$and $|z-w|$ $\leqq \delta$ imply $|F(z, x)-F(w, x)| \leqq \epsilon$ for all $x$. Suppose now that $h \in S^{-}$ and let $\delta=\delta(\epsilon)$. We have $(1-\delta) h \in S$, so that $\Phi_{F}((1-\delta) h)$ is a continuous function, and $\|h-(1-\delta) h\| \leqq \delta$, so that at each $x$ the functions $\Phi_{F}(h)$ and $\Phi_{F}((1-\delta) h)$ differ by at most $\epsilon$. Thus $\Phi_{F}(h)$, being a uni- 
form limit of continuous functions, is continuous. In addition we apply the definition of $\delta(\epsilon)$ to two functions $g$ and $h$ in $S^{-}$and see that $\|g-h\| \leqq \delta(\epsilon)$ implies $\left\|\Phi_{F}(g)-\Phi_{F}(h)\right\| \leqq \epsilon$. That is, $\Phi_{F}$ is uniformly continuous on $S^{-}$.

One extends the inverse mapping $\psi_{F}$ to the closure of the interior of $F$ in the obvious way. Namely, the value of $h=\psi_{F}(g)$ at $x$ is the inverse image under $F(z, x)$ of $g(x)$. One proves as in $\$ 4$ that $h$ is a continuous function. Proceed in much the same way to show that the mapping $\psi_{F}$ is continuous-that is, assume the contrary and deduce a contradiction to the fact that, for each $x, F(z, x)$ is one to one on $K^{-}$. One thus proves

Theorem C. If $F$ is an $H$ simple closed curve in $C(X)$, then $\Phi_{F}$ extends uniquely to a homeomorphism of the closed unit ball onto the closure of the interior of $F$.

\section{REFERENCES}

1. R. Courant, Ueber einige Eigenschaften der Abbildungsfunktionen bei konformer Abbildung, Gött. Nachr., 1914, 101-109.

2. B. W. Glickfeld, Contributions to the theory of holomorphic functions in commutative Banach algebras with identity, Dissertation, Columbia University, New York, 1964.

3. - On the inverse function theorem in commutative Banach algebras, Illinois J. Math. (to appear).

4. E. Hille, Analytic function theory. Vol. II, Introduction to Higher Math., Ginn, Boston, Mass., 1962. MR 34 \#1490.

5. E. R. Lorch, The theory of analytic functions in normed Abelian vector rings, Trans. Amer. Math. Soc. 54 (1943), 414-425. MR 5, 100.

UNIVERSity OF Oregon, Eugene, Oregon 97403 